\title{
A comparative study on allocation/rationing mechanisms operational with/without backorder clearing in divergent supply chains
}

\author{
KURIAN JOHN $^{1, *}$, CHANDRASEKHARAN RAJENDRAN $^{2}$ and HANS ZIEGLER ${ }^{3}$ \\ ${ }^{1}$ Department of Mechanical Engineering, Mar Athanasius College of Engineering, \\ Kothamangalam, Ernakulam 686666, India \\ ${ }^{2}$ Department of Management Studies, Indian Institute of Technology Madras, Chennai 600036, India \\ ${ }^{3}$ Department of Production, Operations and Logistics Management, Faculty of Business Administration and \\ Economics, University of Passau, 94032 Passau, Germany \\ e-mail: kurianjpk2@gmail.com; craj@iitm.ac.in; hans.ziegler@uni-passau.de
}

MS received 7 January 2019; revised 20 June 2019; accepted 4 September 2019

\begin{abstract}
The management of inventory in a divergent supply chain involves inventory allocation/rationing in addition to the determination of order policy parameters. In the case of a stock point feeding product(s) to several downstream members, rationing mechanism can be viewed as a special case of the allocation mechanism. In a supply chain with multi-period ordering cycles, a rationing decision ensures that the entire inventory available with the feeder stock point is rationed to downstream members, whereas an allocation decision need not allocate the entire inventory available, and it is at the discretion of the decision maker at the feeder stock point to retain inventory for possible high priority demands in future periods. In any supply chain permitting backordering of demands from downstream members, the clearing of backorders is a matter of concern. This study addresses the said issue by ensuring that the feeder stock point considers the current period demand for fulfilment only after clearing the backorders with respect to the downstream members. Through this study, an attempt is made to develop mathematical models for supply chains operating with installation-specific costs (holding and shortage) and ordering policy (base stock) over a finite time horizon with and without clearing backorders in the case of rationing as well as allocating inventory to downstream members. Specifically, this work appears to be the first comparative study on allocation and rationing mechanisms in association with/ without backorder clearing mechanisms in divergent supply chains, and their impact on the total supply chain cost.
\end{abstract}

Keywords. Allocation mechanism; rationing mechanism; backorder clearing mechanism; divergent supply chain; base stock; total supply chain cost; mathematical models.

\section{Introduction}

Rationing can be viewed as a special case of allocation, and detailed description is found in the paper by Lagodimos and Koukoumialos [1]. They consider a model comprising of a central stock point feeding several end stock points and in the event of shortages at the central stock point, the central stock point rations all material available to the end stock points. According to the same authors, an allocation decision need not allocate the entire inventory available with the central stock point to the end stock points, but can refrain from doing so, foreseeing critical orders from the end stock points in subsequent periods. In any realistic supply chain, there are retailers which are of priority to the distributor, and such retailers are given preference during occurrences of shortage of products. However, such

*For correspondence

Published online: 04 November 2019 practices could lead to the neglect of retailers with lower priority over longer period of time and this could become detrimental to the performance of any supply chain. A divergent supply chain comprising a manufacturer with sufficient capacity serving a distributor, and the distributor in turn serving a set of retailers is considered in this study. In the following study, the terminology proposed by Lagodimos and Koukoumialos [1] is adopted, referring to rationing as the process ensuring the shipment of all the material available with the distributor to retailers when there is a shortage of material, and allocation as the process that does not necessitate the shipment of the entire inventory available with the distributor to retailers.

In this paper, a model is considered with retailers serving its customers and satisfying the customer demand completely if sufficient inventory is available with the respective retailer; else backordering the unsatisfied demand and shipping this backordered demand on arrival of 
replenishment if any from the distributor. Every retailer immediately communicates the order data to the distributor, as the model assumes zero information lead-time, and the shipment of order takes place if the distributor holds sufficient inventory; else the unsatisfied demand is backlogged by the distributor and is realized on the arrival of replenishment if any from the manufacturer. The distributor places an order to the manufacturer, and it is assumed that the manufacturer is capable of completely satisfying any demand from the distributor in any period during the finite planning horizon. If the distributor holds insufficient inventory to satisfy the retailer demands, the distributor can either ration or allocate the inventory among retailers. Through this study, the main questions to be addressed are:

- Is there any difference between allocation and rationing mechanisms in a divergent supply chain?

- If so, how does it affect the performance of a divergent supply chain in terms of the Total Supply Chain Cost (TSCC)?

- Does the backorder clearing mechanism have an impact on the performance of the supply chain operating with allocation/rationing mechanisms with respect to the TSCC, and does it affect the Total Supply Chain Shortage Cost (TSCSC)?

This technical paper makes an attempt to establish the difference in the above mentioned approaches (rationing/ allocation) when deployed in a divergent supply chain operating with/without backorder clearing mechanism. The results presented as part of this study can be used as a lower bound while solving the problems addressed in this study in a stochastic setting. To the best of our knowledge, it is probably for the first time that such questions are addressed simultaneously in a single research attempt. The organization of this paper is as follows: review of literature is presented in section 2; model and assumptions in section 3; mathematical programming model and solution methodology in section 4; experiments, results and discussions in section 5; and conclusion in section 6 .

\section{Review of literature}

The existing literature in the area of allocation and rationing mechanisms is large, and in this paper a brief discussion on select works is presented. Eppen and Schrage [2] proposed a solution to a depot-warehouse system under the allocation assumption that the incoming order is large enough to obtain an equal probability of stock out at each warehouse. Jönsson and Silver [3] examined a two-level distribution system consisting of a central warehouse supplying a product to branch warehouses and operating with a base stock replenishment policy. In this work, the central warehouse places an order to a supplier having no capacity constraint, and upon receipt of replenishment, the central warehouse allocates all the material to the branch warehouses. The authors explained that this approach can cause the inventories with the branch warehouse to become outof-balance and hence necessitates redistribution. Cao and Silver [4] considered an inventory system consisting of a central warehouse and several regional warehouses. The authors proposed a dynamic heuristic method to determine the appropriate period to perform allocation. Dogru et al [5] considered a two-echelon divergent inventory system with a central stock point serving several downstream stock points. The work makes use of the balance assumption with the aim of aiding the dynamic program in decomposing the problem, and the authors explained that it is the curse of dimensionality that makes the dynamic program hard to decompose. Jackson and Muckstadt [6] formulated the problem of a warehouse serving $N$-retailers for multiple periods and allocating stocks from a robust optimization perspective. The authors claimed that the only motive to hold inventory for future periods, under the setting where the holding cost across the installations is the same, is that of risk pooling. The authors state that the use of dynamic programming to solve this problem requires a state space too large, making it difficult to make practical computations.

Clark and Scarf [7] proposed a rationing policy which tries to attain equal stock out probability for all the end stock points. This rationing policy was later termed as Fair Share (FS) rationing policy. Many researchers have brought out variants of the FS rationing policy; for example, Bollapragada et al [8] and Lagodimos [9]. de Kok [10] proposed the Consistent Appropriate Share (CAS) policy, and the policy tries to maintain a fixed fraction of projected net inventory of every stock point over the system-wide projected net inventory. Many variations of CAS are to be found in the literature. Priority Rationing (PR) policy by Lagodimos [9], Restricted Time Remembering (RTR) policy by Melchiors [11], and Modified Echelon Stock Rationing (MESR) policy by Huang and Iravani [12] are the policies other than FS and CAS found in the literature. Lagodimos and Koukoumialos [1] studied a two-echelon divergent supply chain with a central stock point feeding several end stock points, and making use of periodic review order-up-to policies to control the supply chain. The central stock point rations the available material during the event of shortage at the central stock point. The authors studied a class of rationing rules known as Linear Rationing (LR), which distributes the material to end stock points based on fixed fractions. The authors attempt to develop an alternative definition to LR class rationing function and claim that FS and CAS belong to the LR class. Lagodimos and Koukoumialos [1] provided the definition for rationing and allocation which are followed in this paper. Paul and Rajendran [13] proposed exact and heuristic techniques to solve the problem of inventory optimization in a divergent supply chain. The authors proposed a rationing policy termed as Fractional Rationing (FR) policy, which calculates 
fractions heuristically to arrive at the rationing quantity. Paul and Rajendran [13] use the definition of rationing that Lagodimos and Koukoumialos [1] proposed in their work.

In this study, a backorder clearing mechanism to clear backorders of the distributor at any given period is proposed. The model in this study is such that the distributor considers the current period demand from any retailer for fulfilment only after clearing the backorders of all retailers subject to availability of inventory with the distributor. The clearing of backorders is very essential, and if the distributor does not clear backorders, it could result in backordering of orders from low priority retailers over long periods of time. Backorder clearing mechanism is relevant in supply chains for vaccines, medicines and critical spare parts, to name a few. The backorder clearing mechanism will ensure that no retail outlet or end stock point is neglected in the supply chain when the distributor or the end stock point faces shortage of products, which is very likely in the case of vaccines, drugs and spare parts. The model by Paul and Rajendran [13] does not consider this factor while solving the problem of inventory optimization in a divergent supply chain.

Nahmias and Demmy [14] considered an inventory system for spare parts in air force with low and high priority demands. In their work, the inventory policy consists of a support level, below which all low priority demands are backordered, a reorder point and an order quantity that will ensure certain level of fill rate. They provided solution to a single period model as well as multi period model. Deshpande et al [15] studied a logistic system for U.S. military that manages consumable service parts. The authors classify the demand classes on the basis of arrival rates and shortage costs and propose a static threshold level based rationing policy to manage inventory. The clearing of backorders is on a first-come, first-served basis until inventory level reaches a critical level. According to Möllering and Thonemann [16], customers are clustered into demand classes on the basis of penalty costs or service level requirements. The authors proposed a critical level policy to manage inventory in a divergent system with multiple demand classes for infinite time horizon. The model considered in their study operates with a constant critical level, and in any given period, the backorders of high priority demands from previous periods along with the current period demand of high priority demand class is fulfilled. If the remaining on-hand inventory is above the critical level, then the low priority backorders from previous periods and low priority demands from the current period are fulfilled until the inventory reaches the critical level. The authors claim that optimal backorder clearing is difficult to analyse with conventional methods for periodic and continuous review systems, and made use of a multidimensional Markov chain to model the inventory system. Rajeswari [17] proposed two rationing approaches, one of the rationing approaches proposed by the author distinguished between the backorder and current day's demand, the other rationing approach allows the distributor to have on-hand inventory to co-exist with backlog. The backorder clearing mechanism considered by Rajeswari [17] is different to the one considered in this paper, as in this paper backorders are cleared up to the previous time-period, whereas Rajeswari [17] tried to clear backorders up to present time-period minus the lead time of the distributor. This practise could result in differentiation among backordered demands and hence disparity among downstream members. Along with the difference in backorder clearing mechanism, Rajeswari [17] did not attempt to distinguish between allocation and rationing approaches in divergent supply chains, which is one of the main contributions of this work. Wang et al [18] studied rationing policy in an inventory system with different service criteria for backorders and two demand classes. The authors developed a critical level rationing policy with a threshold mechanism to allocate backorders when multiple backorders are present. They claim this problem to be complex and hence propose heuristics to serve the demand classes with target service levels. Ghosh et al [19] considered an inventory system under continuous review with two demand classes, differentiating them in terms of penalty cost incurred in backordering of demand. The authors proposed a two bin system, and assign separate bins for various demand classes. They claim that this two-bin approach is capable of improving the service levels of the low priority demand classes. Du and Larsen [20] studied reservation policies for advance orders when the chain operates with multiple demand classes. Authors made use of the base-stock policy for managing inventory which issues a replenishment order instantaneously when a customer places an order. They proposed a profit optimization model, and describe expected profit as the difference between expected revenue and expected inventory holding costs. Authors used general reservation policy and claimed that this is capable of improving the results by 5\% in comparison to no reservation and complete reservation. They proposed a simpler reservation method termed "backward delay" which is a method of prioritizing reservation and claimed this method to be easier to perform than general reservation.

The backorder clearing mechanism proposed in this study does not explicitly make use of any priority list in clearing backorders. The mechanism inherently ensures that the demand of the current period is considered for fulfilment by the distributor only after satisfying the backorders of all retailers subject to availability of product. The feeder stock point does not explicitly use a critical level, while making the decision to fulfil backorders unlike the other studies available in the literature. The management of inventory in the supply chain considered in this study is realised by deploying a base stock policy at all the installations, and a backorder clearing mechanism at the distributor. The backorder clearing mechanism inherently ensures the clearance of all the backorders of all retailers by the distributor, subject to availability of sufficient on-hand 
inventory with the distributor. The distributor considers the demand from retailers in the current period for fulfilment only after ensuring that the backorders is cleared, and this approach could enforce the distributor to satisfy the backorders from retailers resulting in increased TSCC; but the actual impact remains to be studied. To the best of our knowledge, various attempts in the area of backorder clearing mechanism make use of a critical level to clear backorders, whereas in this work no such explicit critical level in making the decision to clear backorders is considered. Paul and Rajendran [13] studied a divergent supply chain operating with the objective of minimizing the TSCC, and did not ensure backorder clearing. The objectives of our study are to develop mathematical models (Mixed Integer Linear Program (MILP) models) for the study of supply chains operating with allocation/rationing mechanisms and with/without backorder clearing and to perform a comparative analysis on the performance of the supply chain with the objective of minimizing TSCC over a finite planning horizon.

\section{Model and assumptions}

The supply chain in this study consists of a manufacturer with sufficient production capacity that can fulfill all distributor demand, the distributor serving a set of retailers, and each retailer serving customers with one product. The model does not consider cross docking or transhipment of products among retailers. Over a finite time horizon, multiple periods, each one consisting of one day is studied. All the installations are assumed to operate with base stock policy. During review, an installation will place an order to the upstream installation if the corresponding installation's inventory position is less than the installation's base stock level, in order to raise the inventory position equal to the base stock level. Each retailer faces deterministic, dynamic and discrete external demand from the customers and the sampled customer demands are known a priori over the finite planning horizon.

The distributor operates with/without a backorder clearing mechanism that ensures the clearance of backorders due to all retailers. We do not use any predetermined priority list in clearing the backorders. The distributor considers the demand in the current period for fulfilment only after clearing the backorders due to retailers, and the distributor will either satisfy the retailer demands in the current period completely if sufficient inventory is available with the distributor or else ration/allocate the on-hand inventory and backorder the unmet demand. The mathematical models presented in the next section explain how rationing/allocation is performed and this is enabled through the objective function of minimizing the total supply chain costs. The time taken for any information exchange between stages of the supply chain is assumed to be instantaneous or negligible. In this study, the fixed cost for transportation is assumed to be negligible, and each installation operates with installation specific holding and shortage cost rates associated with base stock policy. Because of the backorder clearing mechanism no backorder of any retailer with respect to the last period is present before the distributor ships any material to any retailer corresponding to today's demand during the present period.

\section{Mathematical programming models and solution methodology}

\subsection{Mathematical formulation: Rationing mechanism with backorder clearing mechanism}

The mathematical formulation is first given for the case of the supply chain operating with the distributor rationing the on-hand inventory to retailers, when there is a shortage of inventory, and the distributor clearing the backorders.

The objective function of the MILP-based mathematical model comprises the sum of holding cost (Total Supply Chain Holding Cost (TSCHC)) and the sum of shortage cost (Total Supply Chain Shortage Cost (TSCHC)) of the supply chain over the entire planning horizon, i.e. $\mathrm{TSCC}=\mathrm{TSCHC}+\mathrm{TSCSC}$ ):

Minimize TSCC $=\sum_{t=1}^{T}\left[\left(h^{D} \times E I_{t}^{D}\right)+\sum_{j=1}^{J}\left(h_{j} \times E I_{j, t}+b_{j} \times B_{j, t}\right)\right]$

For retailers, constraints (2)-(5) have to be obeyed:

It is to be noted that retailer $j$ first receives the replenishment if any, from distributor $D$ on time $t$ that has been shipped on $\left(t-L T_{j}\right)$, and the demand at retailer $j$ is realized after this receipt, if any.

The on-hand inventory of retailer $j$ at the beginning of period $t$ is updated through constraint (2):

$B I_{j, t}=E I_{j, t-1}+Q S_{j, t-L T_{j}}^{D} \quad j=1,2, \ldots, J ; t=1,2, \ldots, T$.

The on-hand inventory of retailer $j$ at the end of period $t$ and the backorder of retailer $j$ at the end of period $t$ are computed using constraint (3):

$$
\begin{gathered}
E I_{j, t}-B_{j, t}=B I_{j, t}-B_{j, t-1}-d_{j, t} \\
j=1,2, \ldots, J ; t=1,2, \ldots, T .
\end{gathered}
$$

The quantity shipped by retailer $j$ to customers is given by constraint (4):

$$
Q S_{j, t}=B I_{j, t}-E I_{j, t} \quad j=1,2, \ldots, J ; t=1,2, \ldots, T .
$$

Constraint (5) states that the demand placed by retailer $j$ to the distributor is equal to the customer demand that retailer $j$ faces in period $t$ : 


$$
D_{j, t}^{D}=d_{j, t} \quad j=1,2, \ldots, J ; t=1,2, \ldots, T .
$$

The constraints for the distributor are given by (6)-(14).

The replenishment lead-time of the distributor, in this study is equal to 1 , and it means that distributor $D$ receives on day $t$, the shipment that has been shipped from manufacturer $M$ on day ( $t-1)$, and the demand at distributor $D$ is realized after this possible receipt.

The on-hand inventory of distributor at the beginning of period $t$ is calculated by constraint (6):

$$
B I_{t}^{D}=E I_{t-1}^{D}+Q S_{D, t-1}^{M} \quad t=1,2, \ldots, T .
$$

Constraint (7) ensures that backorder is cleared before considering the current period demand, and constraint (8) ensures that the quantity backordered by the distributor in period $t$, with respect to retailer $j$, is less than or equal to the demand from retailer $j$ in period $t$ :

$$
Q S_{j, t}^{D}=B_{j, t-1}^{D}+D_{j, t}^{D}-B_{j, t}^{D} \quad j=1,2, \ldots J ; t=1,2, \ldots, T
$$

$$
B_{j, t}^{D} \leq D_{j, t}^{D} \quad j=1,2, \ldots J ; t=1,2, \ldots, T .
$$

The on-hand inventory of the distributor at the end of period $t$, and the backorders corresponding to retailers at the distributor at the end of period $t$ are calculated by (9):

$E I_{t}^{D}-\sum_{j=1}^{J} B_{j, t}^{D}=B I_{t}^{D}-\sum_{j=1}^{J}\left(B_{j, t-1}^{D}+D_{j, t}^{D}\right) \quad t=1,2, \ldots, T$.

Constraints (10) and (11) ensure that the distributor rations the available on-hand inventory to retailers without holding back inventory to satisfy possible future demands from other retailers. These expressions ensure that the mathematical model represents a supply chain that rations the product to the end stock points during the occurrence of shortage.

$$
\begin{gathered}
E I_{t}^{D} \leq \delta_{t} \times M \quad t=1,2, \ldots, T \\
\sum_{j=1}^{J} B_{j, t}^{D} \leq\left(1-\delta_{t}\right) \times M \quad t=1,2, \ldots, T .
\end{gathered}
$$

Constraint (12) ensures that the demand from the distributor in period $t$ is conveyed to the manufacturer:

$$
D_{t}^{M}=\sum_{j=1}^{J} D_{j, t}^{D} \quad t=1,2, \ldots, T .
$$

Constraint (13) prevents the distributor from shipping more than the beginning on-hand inventory to retailers, and constraint (14) ensures that the quantity shipped from the distributor to retailers is equal to the difference between the beginning on-hand inventory and end on-hand inventory of the distributor:

$$
\begin{gathered}
\sum_{j=1}^{J} Q S_{j, t}^{D} \leq B I_{t}^{D} \quad t=1,2, \ldots, T \\
\sum_{j=1}^{J} Q S_{j, t}^{D}=B I_{t}^{D}-E I_{t}^{D} \quad t=1,2, \ldots, T .
\end{gathered}
$$

Constraint (15) ensures that the quantity shipped by the manufacturer is equal to the demand placed by the distributor in period $t$ :

$$
Q S_{D, t}^{M}=D_{t}^{M} \quad t=1,2, \ldots, T .
$$

The initial conditions are as follows:

$$
B_{j, 0}=0 ; \quad j=1,2,3, \ldots, J .
$$

$E I_{0}^{D}=S^{D} . \quad / *$ base stock level of the distributor $* /$

$$
\begin{gathered}
E I_{j, 0}=S_{j} ; j=1,2,3, \ldots, J . \\
/ * \text { base stock level of retailer } \mathrm{j} * / \\
\qquad S_{D, 0}^{M}=0 .
\end{gathered}
$$

$$
\begin{gathered}
Q S_{j, t-L T_{j}}^{D}=0 ; t=1,2,3, \ldots, L T_{j} ; j=1,2,3, \ldots, J . \\
\quad B_{j, 0}^{D}=0 ; \quad j=1,2,3, \ldots, J \\
\delta_{t} \in\{0,1\} ; \quad t=1,2,3, \ldots, T . \\
\text { and all other variables } \geq 0 .
\end{gathered}
$$

\subsection{Mathematical formulation: Allocation mechanism with backorder clearing mechanism}

To develop the mathematical programming model for the supply chain operating with the distributor allocating the product to retailers the Linear Program (LP) relaxation technique is used. Through constraint (22), $\delta_{t}$ is declared as a binary variable, and enforces the distributor to perform rationing when there is a shortage of the product. By relaxing the binary variable to a continuous variable in the interval $[0,1]$, the mathematical model will not enforce the distributor to ration the entire on-hand inventory to retailers during shortage, but will enable the distributor to hold inventory, foreseeing the possible critical demands from retailers in future periods. Basically, the constraints (10) and (11) are made redundant and inactive. Note that, in enforcing the allocation mechanism, the MILP model inherently performs the task of allocating the on-hand inventory with respect to the present and future demands of retailers through the lookahead feature of the mathematical model that has information about demand of retailers over the entire planning horizon. 
The binary variable ensures that the variable representing the on-hand inventory of the distributor at the end of period $t$ and the variable representing the total backorder at the distributor corresponding to all retailers at the end of period $t$ do not coexist. However, by preventing the coexistence of these variables, it is ensured that the mathematical model remains a rationing model. When the binary variable is relaxed and converted to a continuous variable in the $[0,1]$ interval, the mathematical model will not guarantee the prevention of the coexistence of variables representing the backorder at the distributor and on-hand inventory at the distributor. Hypothetical examples are presented in Appendix-A and Appendix-B to highlight the difference between the models operating with and without backorder clearing mechanism, and between rationing and allocation approaches respectively. In the mathematical model of the supply chain with the distributor performing allocation during shortage of product, all constraints (except constraint (22), now replaced by $\left.0 \leq \delta_{t} \leq 1\right)$ remain the same as in the mathematical model of the supply chain with the distributor performing the rationing mechanism.

\subsection{Mathematical formulation: Rationing mechanism without backorder clearing mechanism}

Constraint (8) enforce the distributor to clear backorders corresponding to retailers before consideration of the present period demand from retailers by the distributor. By removing this constraint (constraint (8)), the mathematical model presented in subsection 4.2 is converted to the mathematical model of the supply chain rationing the inventory during shortage and enforcing no backorder clearing mechanism.

\subsection{Mathematical formulation: Allocation mechanism without backorder clearing mechanism}

By removing constraint (8), and relaxing the binary variable $\delta_{t}$ (in constraint (22)) to $0 \leq \delta_{t} \leq 1$, i.e. a continuous variable, in the mathematical model in subsection 4.2 , the mathematical model of the supply chain rationing and clearing backorders is converted to the mathematical model of the supply chain allocating and not enforcing the clearance of backorders.

\section{Experiments, results and discussions}

\subsection{Design of the experiments}

The experiments are executed with specific values for the various parameters with the objective of testing the performance of the mathematical programming model. In this study, two scenarios are considered: first, all retailers are assumed to be similar, and second, the retailers are separated into different classes with different shortage cost rates. Several studies in the literature report the use of shortage cost rates to segregate retailers into demand classes; Dogru et al [5], Deshpande et al [15], and Möllering and Thonemann [16] are some examples.

In the experiment, the number of retailers is set to 4, i.e. $J=4$. Cost Setting-1 (CS-1) represents the case of retailers being similar with the same holding and shortage cost-rate across retailers. The parameters for CS-1 are: $h_{1}=h_{2}=h_{3}=h_{4}=2 ; b_{1}=b_{2}=b_{3}=b_{4}=10 ; h^{D}=1$; $L T_{1}=L T_{2}=L T_{3}=L T_{4}=1 ; T=250 ; M=500000$. The review period for the experiment is fixed as one day. Cost Setting-2 (CS-2) represents the case of retailers being dissimilar, with the same holding cost-rate but different shortage cost-rates across retailers. The parameters for CS2 are $h_{1}=h_{2}=h_{3}=h_{4}=2 ; b_{1}=40 ; b_{2}=30 ; b_{3}=20$; $b_{4}=10 ; h^{D}=1 ; L T_{1}=L T_{2}=L T_{3}=L T_{4}=1 ; T=250$; $M=500000$. By setting the shortage cost rates of retailers as shown above, demand classes are separated. The first retailer, having the highest shortage cost-rate, represents the retailer with high priority demand class, and the fourth retailer with the lowest shortage cost-rate represents the retailer with the lowest priority demands.

The customer demand placed to retailer $j$ in period $t$ is sampled a priori over the finite planning horizon. It is assumed that the input demand follows uniform distribution, as Daniel and Rajendran [21] state that the use of uniform distributions results in the generation of hard test bed problems. The customer demands faced by retailers are set to a lower limit and an upper limit, and for Demand Setting- $A$ (DS-A) the limits are 0 and 2000 , i.e. $d_{j, t} \forall j ; \forall t$, for DS-A is within (0, 2000). For Demand Setting-B (DS-B), the lower limits and upper limits for sampling the customer demands from uniform distribution, for the entire time horizon, are: $(0,2000),(0,1500),(0,1000),(0,500)$ respectively for retailers $j=1$ to 4 ; and for Demand Setting$C$ (DS-C) the lower limits and upper limits for sampling the customer demands from uniform distribution, for the entire time horizon, are: $(0,500),(0,1000),(0,1500),(0,2000)$ respectively for retailers $j=1$ to 4 . The mathematical programming model is solved using four demand streams (two first runs and corresponding antithetic runs), and the average costs are reported. For determining the optimal solutions IBM ILOG CPLEX Optimization Studio solver and a computer with 32 bit, Intel(R) Core(TM) i5-2400QM CPU @3.10GHz processor and 2 GB RAM have been used.

\subsection{Results of the experiments}

The results of the experiments with respect to Total Supply Chain Shortage Cost (TSCSC), Total Supply Chain Holding Cost (TSCHC) and Total Supply Chain Cost (TSCC) of the supply chain with a distributor performing rationing/ allocation of on-hand inventory with backorder clearing 
mechanism in both cases are shown in figure 1 for the two cost and the three demand settings. In figure 2 the comparison between allocation mechanism and rationing mechanism in divergent supply chains operating without clearing the backorders is depicted. As expected, the performance of the allocation mechanism in terms of TSCC is superior to the rationing mechanism for all the demand settings and cost settings. Figure 3 displays the comparison between the performances of the supply chain rationing the inventory during shortage and operating with and without clearing backorders. Figure 4 shows the comparison between the performances of the supply chain allocating the inventory and operating with and without clearing backorders.

\subsection{Discussion of the results with focus on the TSCC}

From figures 1 and 2 it can be observed that the TSCC of the supply chain, comprising holding and shortage costs, is consistently the lowest when the supply chain operates with the distributor allocating inventory to end stock points. The performance of the supply chain operating with allocation mechanism can be attributed to the nature of the mathematical model enabling the solver to foresee demands in future periods or the ability to see the demands over the entire planning horizon and accordingly make shipments to minimize the TSCC. The allocation mechanism allows the distributor to withhold inventory for possible future shipments to retailers, and hence the solver is flexible in deciding the shipment quantity to retailers; whereas the rationing mechanism ensures that the distributor ships the entire inventory during shortage, and hence the solver does not inherently allow for withholding of inventory by foreseeing future demands. However, a supply chain operating with rationing mechanism is closer to real life as it may not be possible to have information about future period demands.

\subsection{Discussion of the results with focus on the TSCSC}

The cost-wise breakdown of TSCC in terms of the average TSCSC and average TSCHC can be seen for various settings in all of the figures. The capability of the backorder clearing mechanism in reducing the average TSCSC (across experimental settings) in the supply chain operating with the distributor rationing the on-hand inventory to retailers in comparison to a rationing system not clearing backorders is observable from figure 3 presented in the previous section. From the left half of figure 3 , it is observable that, the average TSCSC for all the demand settings of CS-1 for the supply chain operating with backorder clearing mechanism while rationing inventory is lower than the case where no backorder clearing is enforced. From the right half of figure 3, it is observable that for all the demand settings of CS-2 for the supply chain rationing inventory and clearing backorders the

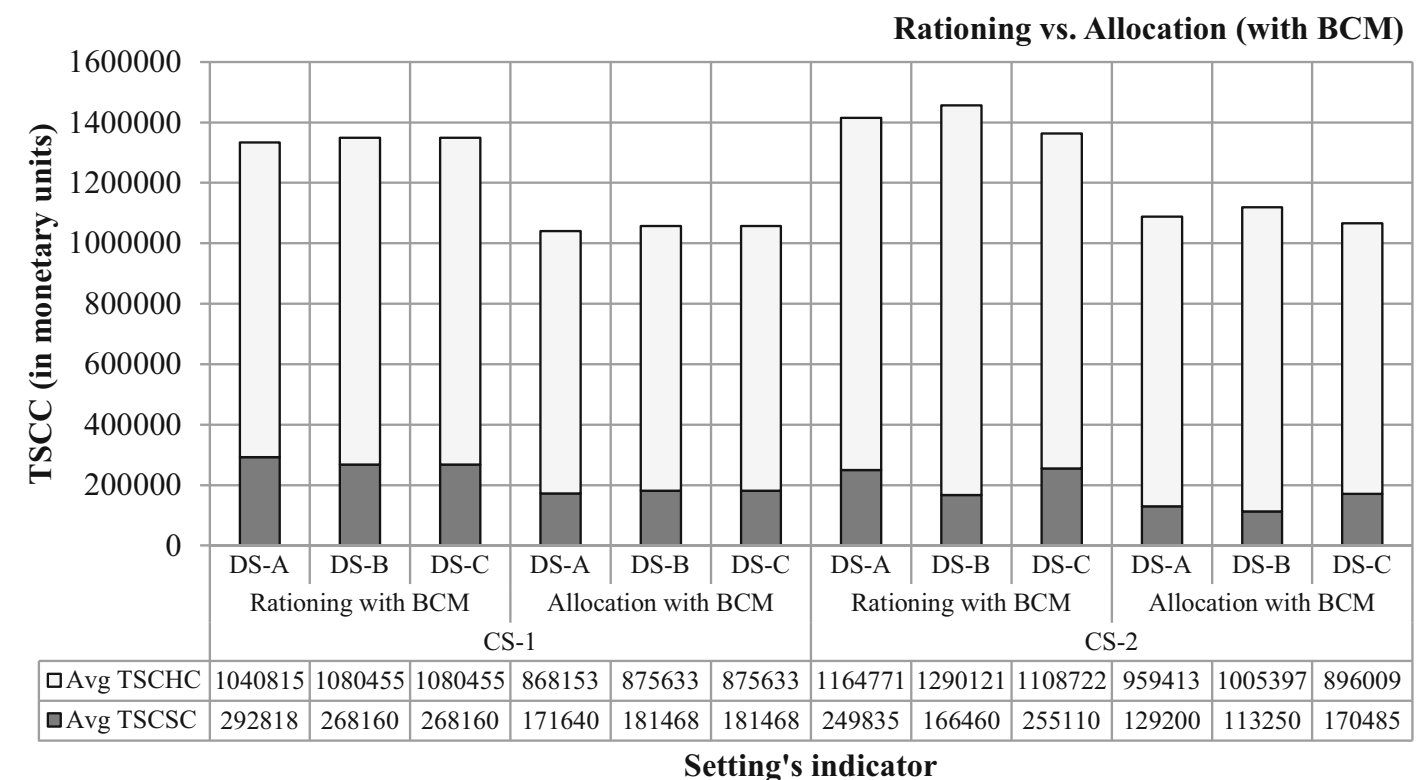

Legends: TSCC: Total Supply Chain Cost; TSCHC: Total Supply Chain Holding Cost; TSCSC: Total Supply Chain Shortage Cost; BCM: Backorder Clearing Mechanism; DS-A: Demand Setting-A; DS-B: Demand Setting-B; DS-C: Demand SettingC; CS-1: Cost Setting-1; CS-2: Cost Setting-2.

Figure 1. Comparison in terms of costs of supply chain operating with rationing mechanism vs. supply chain operating with allocation mechanism, both with backorder clearing mechanism (BCM). 


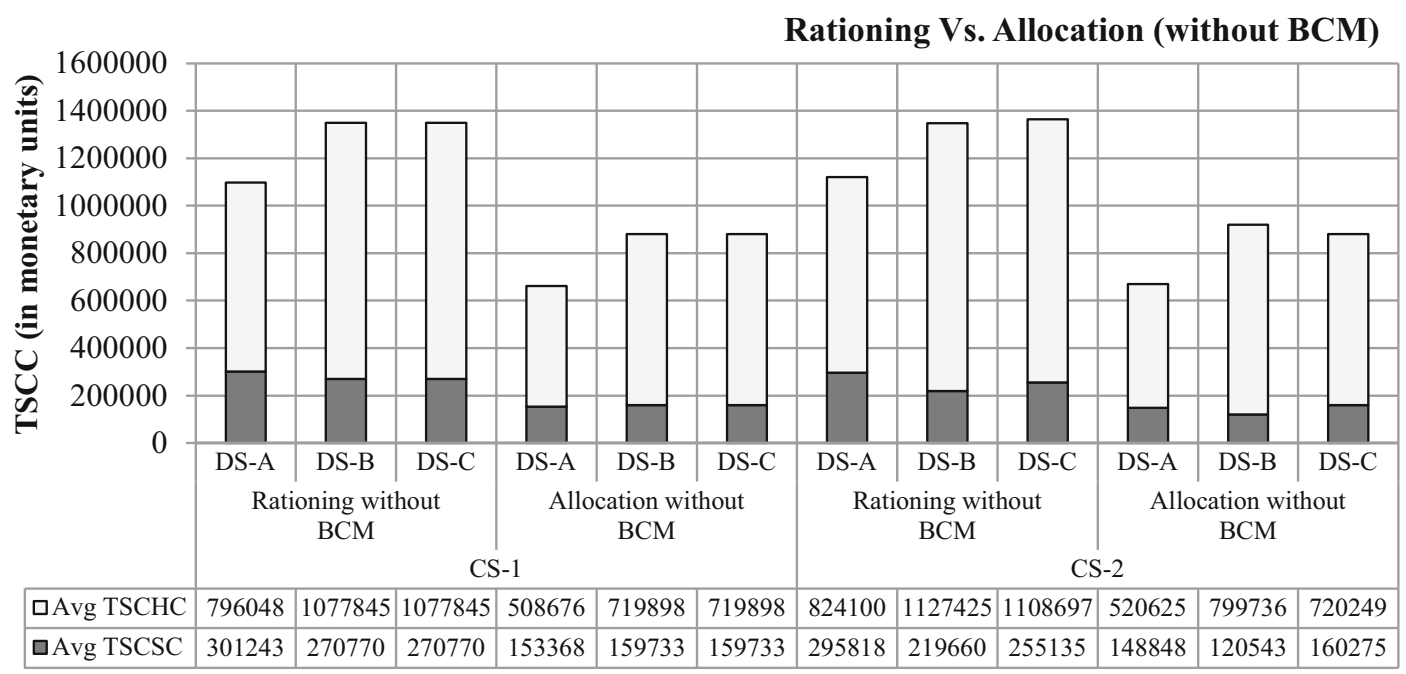

\section{Setting's indicator}

Legends: TSCC: Total Supply Chain Cost; TSCHC: Total Supply Chain Holding Cost; TSCSC: Total Supply Chain Shortage Cost; BCM: Backorder Clearing Mechanism; DS-A: Demand Setting-A; DS-B: Demand Setting-B; DS-C: Demand SettingC; CS-1: Cost Setting-1; CS-2: Cost Setting-2.

Figure 2. Comparison in terms of costs of supply chain operating with rationing mechanism vs. supply chain operating with allocation mechanism, both without backorder clearing mechanism (BCM).

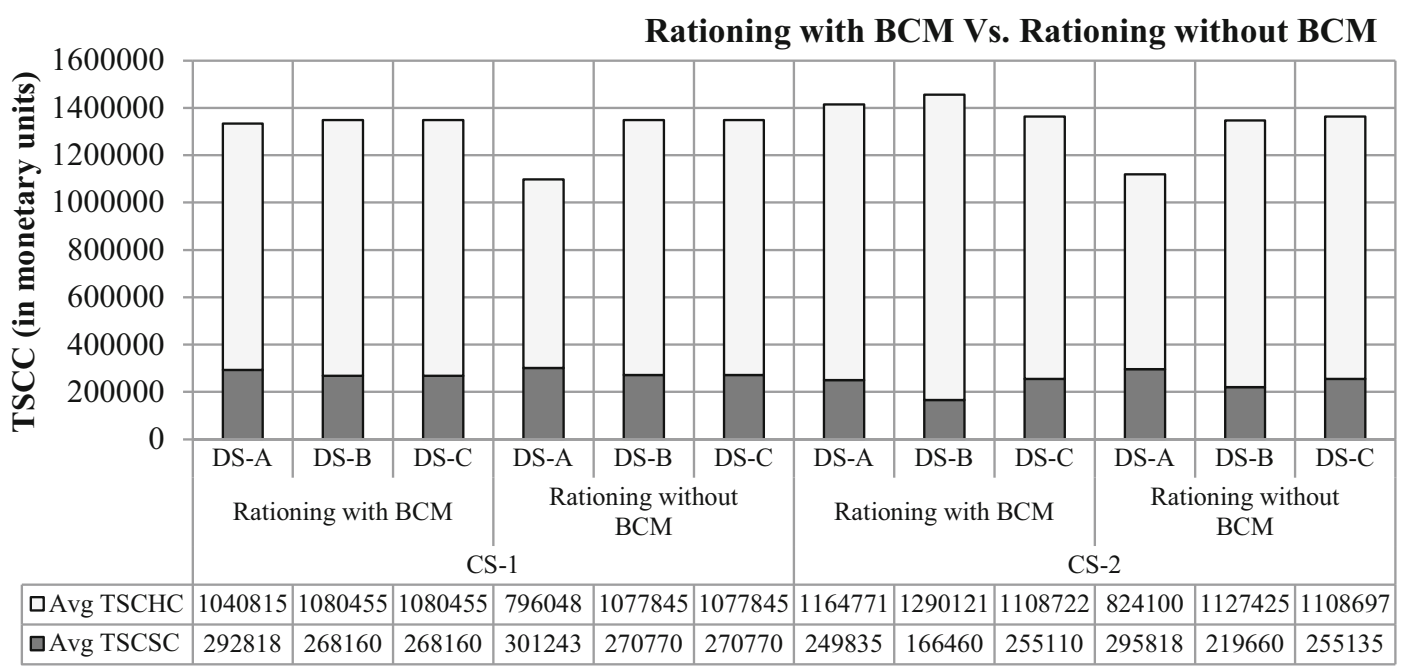

\section{Setting's indicator}

Legends: TSCC: Total Supply Chain Cost; TSCHC: Total Supply Chain Holding Cost; TSCSC: Total Supply Chain Shortage Cost; BCM: Backorder Clearing Mechanism; DS-A: Demand Setting-A; DS-B: Demand Setting-B; DS-C: Demand SettingC; CS-1: Cost Setting-1; CS-2: Cost Setting-2.

Figure 3. Comparison in terms of costs of supply chain operating with rationing mechanism and backorder clearing mechanism (BCM) vs. supply chain operating with rationing mechanism without BCM.

TSCSC is lower than the case where the backorders are not cleared before considering the demand for the current time-period. So, from the results it can be inferred that in a supply chain that rations inventory during shortage, backorder clearing mechanism can be deployed to reduce the shortage costs incurred, and hence could result in improving the fill rates of low priority retailers.
It is observable from figure 4 (left side) that the backorder clearing mechanism in the supply chain operating with the distributor allocating the on-hand inventory to retailers does not reduce the average TSCSC for cost setting-1 across all demand settings. Cost setting- 1 represents the case where all the retailers are similar as they have similar backorder cost-rates. The reason that the average TSCSC 


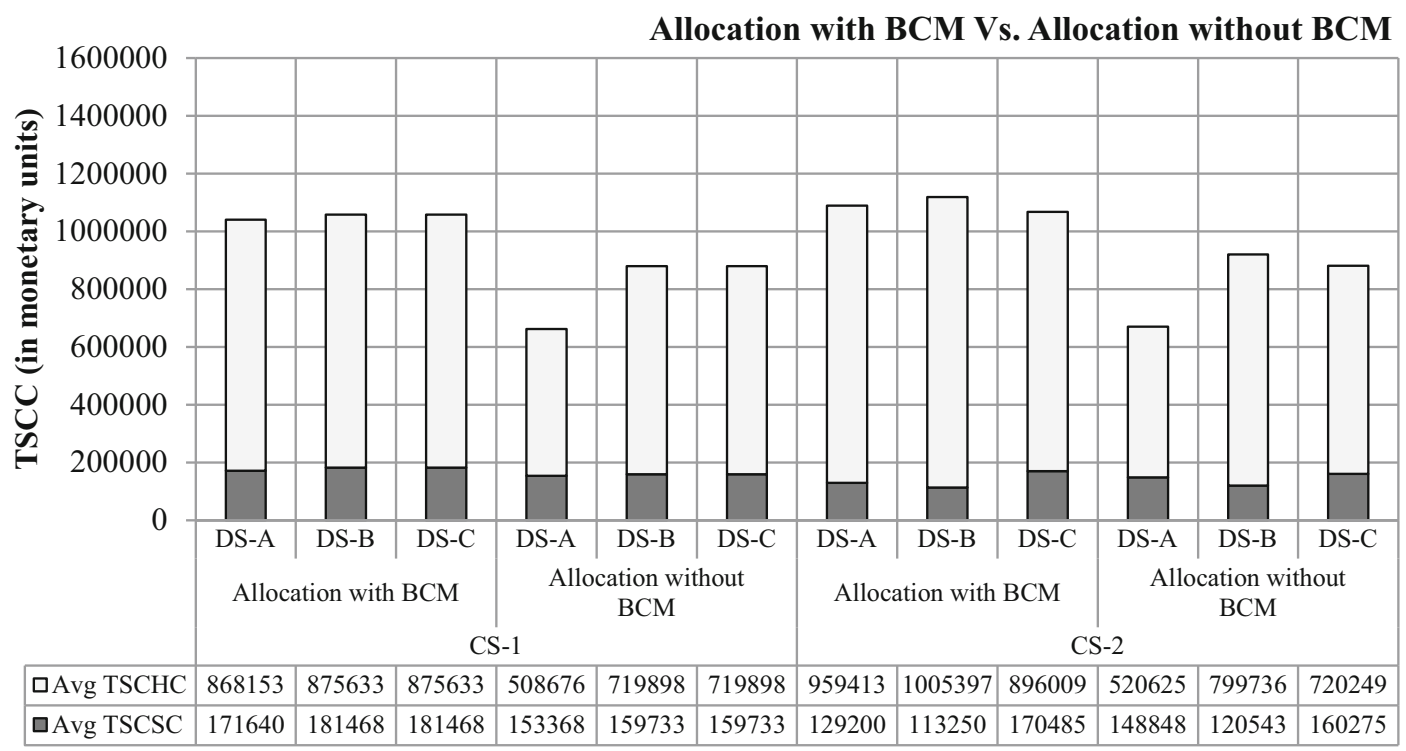

\section{Setting's indicator}

Legends: TSCHC: Total Supply Chain Holding Cost; TSCSC: Total Supply Chain Shortage Cost; BCM: Backorder Clearing Mechanism; DS-A: Demand Setting-A; DS-B: Demand Setting-B; DS-C: Demand Setting-C; CS-1: Cost Setting-1; CS-2: Cost Setting-2.

Figure 4. Comparison in terms of costs of supply chain operating with allocation mechanism and backorder clearing mechanism $(\mathrm{BCM})$ vs. supply chain operating with allocation mechanism without BCM.

does not reduce by enforcing backorder clearing mechanism is due to the fact that an allocation decision represents an ideal situation in the case of a supply chain when retailers are similar. As allocation decisions are intractable and the suitability for products such as vaccines, critical spare parts and important medicines is low, these results can be used as a lower bound while modelling the supply chain on realistic lines.

The scenario captured by cost setting- 2 represents the situation where the retailers are dissimilar, and dissimilarity between retailers is captured by setting their cost-rates as dissimilar (refer section 5.1). From figure 4 (right side) it is observable that the average TSCSC for DS-A and DS-B for CS-2 is lower for the situation when backorders are cleared while allocating inventory. This is due to the fact that the retailers are treated with different priorities and backorder clearing in such a scenario can help reduce the TSCSC of the supply chain. In such scenarios where there are dissimilar retailers, backorder clearing can be deployed as a strategy to bring down overall shortage costs of the supply chain and hence improve the fill rates of low priority retailers. In the situation where CS-2 is deployed along with DS-C, the results do not adhere to the above mentioned pattern and are attributable to the combination of the cost setting and the demand setting which being unrealistic, but executed to test the performance of the mathematical model. In DS-C the retailer demands are sampled from: $(0,500),(0,1000),(0$, $1500),(0,2000)$ for retailer- 1 to retailer- 4 respectively and, the cost-rates are set as: $b_{1}=40 ; b_{2}=30 ; b_{3}=20 ; b_{4}=$ 10 for retailer- 1 to retailer- 4 respectively. This kind of a setting is unlikely because, if the first retailer is considered, the demand is sampled between 0 and 500 and the backlog cost-rate is set as 40 and, for the last retailer the demand is sampled between 0 and 2000 and the backlog cost-rate is set as 10. This kind of a setting is highly unlikely in real scenarios as the retailer given highest priority would be the retailer buying maximum quantity, and this setting defies that logic, and is presented in this study only to test the performance of the mathematical model under such situations. Future research could extend the present study by considering lost sales, ordering costs and stochastic demands (see John et al [22] and John et al [23] for details).

\section{Conclusion}

In this paper, a supply chain operating with a manufacturer supplying a product to a distributor, the distributor serving a set of retailers, and retailers in turn serving the customers has been considered. The distributor rations/allocates the on-hand inventory to retailers when the shortage of product occurs in a given period. All the members operate with base stock policy.

A backorder clearing mechanism to clear the retailer orders backordered by the distributor is proposed and a mathematical programming model of the supply chain with the objective of minimizing the TSCC comprising of holding and shortage costs is presented. The performance of a divergent supply chain with rationing/allocation mechanism with/without backorder clearing mechanism with respect to TSCC is analysed. It is observable from the 
results that the performance of the supply chain operating with the distributor allocating on-hand inventory to retailers and no backorder clearing mechanism in terms of TSCC is consistently superior irrespective of the experiment setting. This superior performance of the supply chain operating with the distributor allocating on-hand inventory to retailers and not clearing the backorders can be attributed to: (1) the knowledge of customer demands over the planning horizon, and (2) the freedom to withhold inventory to satisfy these possible future demands. However, supply chains of this nature, capable of predicting future customer demands with minimal error, is unrealistic. Hence, this kind of supply chain models represents an idealistic scenario, and the corresponding solution is a lower bound on the objective function of minimizing the TSCC for supply chains operating with the assumptions of rationing mechanism without backordering the retailer demands.

The ability of the backorder clearing mechanism in reducing the TSCSC, for all of the settings of the supply chain operating with rationing mechanism, is observable from the results of the experiments. The performance of the supply chain operating with the distributor allocating the on-hand inventory to retailers during shortage and not clearing its backorders is superior in most of the experiment settings, but represents an ideal case, and hence this can be viewed as a lower bound on the objective function of minimizing TSCC in comparison to the realistic supply chain operating with rationing mechanism. The results presented as part of this study can be used as a lower bound while solving the problems addressed in this study in stochastic setting. The backorder clearing mechanism proposed in this study will enforce the distributor to satisfy the backorders subject to availability of products, thereby reducing TSCSC, and could result in higher TSCC due to increase in TSCHC.

\section{Acknowledgement}

This research work has been carried out with the financial support from IIT Madras, University of Passau and DAAD as a part of the joint $\mathrm{PhD}$ degree programme. The authors are thankful to reviewers and the Editor for their suggestions and comments to improve the earlier version of the paper.

\section{Appendix}

\section{Terminology}

\section{Parameters}

$j \quad$ retailer index where $j=1,2,3, \ldots, J$

$J \quad$ total number of retailers

$h_{j} \quad$ holding cost-rate of retailer $j$

$b_{j} \quad$ shortage (backorder) cost-rate of retailer $j$

$h^{D} \quad$ holding cost-rate of the distributor
$L T_{j} \quad$ replenishment lead-time of retailer $j$

$T$ number of periods considered in the model

$M \quad$ a large positive value, associated with the use of a $\{0,1\}$ binary variable

$d_{j, t} \quad$ customer demand placed to retailer $j$ in period $t$

\section{Decision variables}

$B I_{j, t} \quad$ on-hand inventory of retailer $j$ at the beginning of period $t$

$B I_{t}^{D} \quad$ on-hand inventory of distributor at the beginning of period $t$

$E I_{j, t} \quad$ on-hand inventory of retailer $j$ at the end of period

$$
t
$$

$E I_{t}^{D} \quad$ on-hand inventory of the distributor at the end of period $t$

$B_{j, t} \quad$ backorder of retailer $j$ at the end of period $t$

$D_{j, t}^{D} \quad$ demand placed to the distributor by retailer $j$ in period $t$

$D_{t}^{M} \quad$ demand placed to the manufacturer in period $t$

$Q S_{j, t}^{D} \quad$ quantity shipped from the distributor to retailer $j$ in period $t$

$Q S_{j, t} \quad$ quantity shipped from retailer $j$ to customer in period $t$

$Q S_{D, t}^{M} \quad$ quantity shipped from the manufacturer to the distributor in period $t$

$S_{j} \quad$ base stock level of retailer $j$

$S^{D} \quad$ base stock level of the distributor

$\delta_{t} \quad$ binary variable to prevent the co-existence of onhand inventory and backorder at the distributor in period $t$

TSCC total supply chain cost over all installations and periods

$B_{j, t}^{D} \quad$ backorder at the distributor with respect to retailer $j$ at the end of period $t$ (i.e., unmet demand of retailer $j$ in period $t$; does not include backorder, if any, in periods prior to period $t$ )

\section{Appendix A}

An illustrative example is presented to establish the difference in functioning of the supply chain when the distributor operates with backorder clearing mechanism and when the distributor does not first clear the backorders of the previous day. Consider a supply chain operating with two retailers, a distributor, and a manufacturer. For the symbols, refer to subsection 4.1.

$$
\begin{aligned}
& \text { Parameters: } h_{1}=h_{2}=2 ; b_{1}=10 ; b_{2}=40 ; \\
& \begin{aligned}
d_{1,1}=10 ; d_{2,1}=15 ; d_{1,2}=15 ; d_{2,2}=30 ; d_{1,3}=25 ; d_{2,3} \\
\quad=25
\end{aligned}
\end{aligned}
$$

$/ *$ note $D_{j, t}^{D}=d_{j, t}$, recall constraint (5) in subsection 4.2 for understanding $* /$ 
We set the decision variable $S^{D}=20$.

It can be observed from the above example that on day-2 $Q S_{1,2}^{D}=5+15-15=5$ and $Q S_{2,2}^{D}=0+30-$ $10=20$ when the distributor clears the backorders, whereas in the model with no clearing of backorders $Q S_{1,2}^{D}=0$ and $Q S_{2,2}^{D}=25$ due to $Q S_{1,2}^{D}+Q S_{2,2}^{D} \leq B I_{2}^{D}$, and $b_{2}>b_{1}$.

\section{Appendix B}

An illustrative example to demonstrate the difference between allocation approach and rationing approach is presented. See expressions (9), (10), (11), and (22). In the mathematical model of the supply chain with the distributor rationing the on-hand inventory to retailers, the distributor is enforced to perform rationing mechanism through expressions (10) and (11), and (22). The computation of these expressions in a hypothetical setting on day $t$ with rationing mechanism is as follows:

\begin{tabular}{ll}
\hline $\begin{array}{l}\text { With backorder clearing } \\
\text { mechanism: }\end{array}$ & $\begin{array}{c}\text { Without backorder } \\
\text { clearing mechanism: }\end{array}$ \\
\hline Day-1: & Day-1: \\
$B I_{1}^{D}=20$ & $B I_{1}^{D}=20$ \\
$\left(Q S_{j, t}^{D}=B_{j, t-1}^{D}+D_{j, t}^{D}-B_{j, t}^{D}\right)$, & - \\
and $\left(B_{j, t}^{D} \leq D_{j, t}^{D}\right)$ & \\
$Q S_{1,1}^{D}=0+10-5=5$ & $Q S_{1,1}^{D}=5$ \\
$Q S_{2,1}^{D}=0+15-0=15$ & $Q S_{2,1}^{D}=15$ \\
$B_{1,1}^{D}=5 ; B_{2,1}^{D}=0$ & $B_{1,1}^{D}=5 ; B_{2,1}^{D}=0$ \\
$E I_{1}^{D}=0 ; D_{1}^{M}=25$ & $E I_{1}^{D}=0 ; D_{1}^{M}=25$ \\
Day-2: & Day-2: \\
$B I_{2}^{D}=25$ & $B I_{2}^{D}=25$ \\
$Q S_{1,2}^{D}=5+15-15=5$ & $Q S_{1,2}^{D}=0\left(\because Q S_{1,2}^{D}\right.$ \\
& $\left.+Q S_{2,2}^{D} \leq B I_{2}^{D}, b_{2}>b_{1}\right)$ \\
$Q S_{2,2}^{D}=0+30-10=20$ & $Q S_{2,2}^{D}=25\left(\cdot Q S_{1,2}^{D}\right.$ \\
& $\left.+Q S_{2,2}^{D} \leq B I_{2}^{D}, b_{2}>b_{1}\right)$ \\
$B_{1,2}^{D}=15 ; B_{2,2}^{D}=10$ & $B_{1,2}^{D}=20 ; B_{2,2}^{D}=5$ \\
$E I_{2}^{D}=0 ; D_{2}^{M}=45$ & $E I_{2}^{D}=0 ; D_{2}^{M}=45$ \\
Day-3: $_{B I_{3}^{D}=45}$ & $\mathbf{D a y - 3 :}$ \\
$Q S_{1,3}^{D}=15+25-25=15$ & $B I_{3}^{D}=45$ \\
$Q S_{2,3}^{D}=10+25-5=30$ & $Q S_{1,3}^{D}=15\left(\cdot Q S_{1,3}^{D}\right.$ \\
& $\left.+Q S_{2,3}^{D} \leq B I_{3}^{D}, b_{2}>b_{1}\right)$ \\
$B_{1,3}^{D}=25 ; B_{2,3}^{D}=5$ & $Q S_{2,3}^{D}=30\left(\because Q S_{1,3}^{D}\right.$ \\
$E I_{3}^{D}=0$ & $\left.+Q S_{2,3}^{D} \leq B I_{3}^{D}, b_{2}>b_{1}\right)$ \\
& $B_{1,3}^{D}=30 ; B_{2,3}^{D}=0$ \\
& $E I_{3}^{D}=0$ \\
&
\end{tabular}

$$
\begin{array}{r}
E I_{t}^{D}-\sum_{j=1}^{J} B_{j, t}^{D}=B I_{t}^{D}-\sum_{j=1}^{J}\left(B_{j, t-1}^{D}+D_{j, t}^{D}\right) \\
50-0=100-(20+30) ;
\end{array}
$$

i.e., the solver is capable of enforcing the value 50 for the variable $E I_{t}^{D}$ because of expressions (10), (11), and (22), as this can be observed from the computations shown below, with $M$ set as 500000 .

$$
\begin{gathered}
E I_{t}^{D} \leq \delta_{t} \times M \\
50 \leq 1 \times 500000 ; \\
\sum_{j=1}^{J} B_{j, t}^{D} \leq\left(1-\delta_{t}\right) \times M \\
0 \leq(1-1) \times 500000 ;
\end{gathered}
$$

With $\delta_{t} \in\{0,1\}$. Relaxation of the constraint (22), with $\delta_{t}$ being a continuous variable in the $[0,1]$ interval will allow the coexistence of $E I_{t}^{D}$ and $\sum_{j=1}^{J} B_{j, t}^{D}$, and convert the mathematical model of the supply chain (operating with the distributor rationing the on-hand inventory during shortage) to an LP model the supply chain with the distributor allocating the on-hand inventory. The computation of constraint (9) is as follows:

$$
\begin{gathered}
E I_{t}^{D}-\sum_{j=1}^{J} B_{j, t}^{D}=B I_{t}^{D}-\sum_{j=1}^{J}\left(B_{j, t-1}^{D}+D_{j, t}^{D}\right) \\
100-50=100-(20+30) ; \\
E I_{t}^{D} \leq \delta_{t} \times M \\
100 \leq 0.5 \times 500000 ; \\
\sum_{j=1}^{J} B_{j, t}^{D} \leq\left(1-\delta_{t}\right) \times M \\
50 \leq(1-0.5) \times 500000 ;
\end{gathered}
$$

with $0 \leq \delta_{t} \leq 1$. Where an example out of many values possible for $E I_{t}^{D}$ and $\sum_{j=1}^{J} B_{j, t}^{D}$ is used to show the difference between the two approaches. The LP model allows for the co-existence of $E I_{t}^{D}$ and $\sum_{j=1}^{J} B_{j, t}^{D}$ in order to allocate this onhand inventory in future periods, so as to reduce the total supply chain cost, especially reducing the future shortage costs with respect to those retailers having higher shortage cost rates.

\section{References}

[1] Lagodimos A G and Koukoumialos S 2008 Service performance of two-echelon supply chains under linear rationing. International Journal of Production Economics 112: 869-884

[2] Eppen G and Schrage L 1981 Centralized ordering policies in a multi-warehouse system with lead times and random 
demand. TIMS Studies in the Management Sciences 16: $51-67$

[3] Jönsson H and Silver E A 1987 Analysis of a two-echelon inventory control system with complete redistribution. Management Science 33: 215-227

[4] Cao D and Silver E A 2005 A dynamic allocation heuristic for centralized safety stock. Naval Research Logistics 52: 513-526

[5] Dogru M K, de Kok A G and van Houtum G J 2009 A numerical study on the effect of the balance assumption in one-warehouse multi-retailer inventory systems. Flexible Services and Manufacture Journal 21: 114-147

[6] Jackson P L and Muckstadt J A 2015 Multi-period stock allocation via robust optimization. ORIE Technical ReportsCornell University http://hdl.handle.net/1813/39275

[7] Clark A J and Scarf H 1960 Optimal policies for multiechelon inventory problem. Management Science 6: 475-490

[8] Bollapragada S, Akella R and Srinivasan R 1999 Centralized ordering and allocation policies in a two-echelon system with non-identical warehouses. European Journal of Operational Research 106: 74-81

[9] Lagodimos A G 1992 Multi-echelon service models for inventory systems under different rationing policies. International Journal of Production Research 30: 939-958

[10] de Kok A G 1990 Hierarchical production planning for consumer goods. European Journal of Operational Research 45: 55-69

[11] Melchiors P 2003 Restricted time-remembering policies for the inventory rationing problem. International Journal of Production Economics 81: 461-468

[12] Huang B and Iravani S M R 2007 Optimal production and rationing decisions in supply chains with information sharing. Operations Research Letters 35: 669-676

[13] Paul B and Rajendran C 2010 Optimal and heuristic basestock levels and rationing policy for a divergent supply chain. International Journal of Industrial and Systems Engineering 5: 460-484
[14] Nahmias S and Demmy W S 1981 Operating characteristics of an inventory system with rationing. Management Science 27: $1236-1245$

[15] Deshpande V, Cohen M A and Donohue K 2003 A threshold inventory rationing policy for service-differentiated demand classes. Management Science 49: 683-703

[16] Möllering K T and Thonemann U W 2008 An optimal critical level policy for inventory systems with two demand classes. Naval Research Logistics 55: 632-642

[17] Rajeswari S 2009 Rationing approaches and order-up-to level policy in a two-stage divergent supply chains Unpublished M.S. Thesis, Department of Management Studies, Indian Institute of Technology Madras, Chennai, India

[18] Wang D, Tang O and Huo J 2013 A heuristic for rationing inventory in two demand classes with backlog costs and a service constraint. Computers \& Operations Research 40: 2826-2835

[19] Ghosh S, Piplani R and Viswanath S 2015 A new two-bin policy for inventory systems with differentiated demand classes. Production and Operations Management Production and Operations Management Society 24: 840-850

[20] Du B and Larsen C 2017 Reservation policies of advance orders in the presence of multiple demand classes. European Journal of Operational Research 256: 430-438.

[21] Daniel S R J and Rajendran C 2005 A simulation-based genetic algorithmic approach to inventory optimization in a serial supply chain. International Transactions in Operational Research 12: 101-127

[22] John K, Rajendran C and Ziegler H 2016 Investigation of order-up-to policy and allocation-rationing mechanism for divergent supply chains with multiple objectives In: Ravindran A R (Ed.) Multiple Criteria Decision Making in Supply Chain Management U.S.A. CRC Press

[23] John K, Rajendran C and Ziegler H 2017 Investigations on divergent supply chains operating with backorder clearing mechanisms, multiple objectives and order policies Unpublished Ph.D. Thesis, Department of Management Studies, Indian Institute of Technology Madras Chennai, India and University of Passau Germany 